\title{
MẠNG TÍNH TOÁN VÀ ƯNG DỤNG
}

\author{
HOÀNG KIẾM, ĐỖ VĂN NHON
}

\begin{abstract}
In this paper we propose a model that can be used for representing knowlege. It is called a computational net. We also discuss some on a computational net, and prove the algrorithms used to solve the problems. Algorithms have been implemented and tested carefully in various applications. In this paper we bring out two application examples in geometry and in chemistry.
\end{abstract}

\section{GIÓI THIỆU}

Một trong những vấn đề hiện nay đang được quan tâm của "Trí Tuệ Nhân Tạo" là nghiên cứu các phương pháp biểu diễn và xử lý tri thức. Trên cơ sở đó có thể tạo ra những chương trình "thông minh" ở một mức độ nào đó. Trong nhiều lĩnh vực chúng ta thường gặp những vấn đề đặt nra dưới dạng như sau: Chúng ta phải thực hiện những tính toán hay suy diễn ra những yếu tố cần thiết nào đó từ một số yếu tố đã được biết trước. Để giải quyết vấn đề người ta phải vận dụng một số hiểu biết (tri thức) nào đó về những liên hệ giữa các yếu tố đang được xem xét. Những liên hệ cho phép ta có thể suy được một số yếu tố giả thiết đã biết một số yếi tố khác. Trong [1] và [4] có nêu lên một bài toán trong toán học, vật lý, hóa học có dạng như trên.

Trong bài báo này chúng tôi đưa ra một mô hình biểu diễn và xử lý tri thức có thể áp dụng giải tự động các bài toán trên, và ta gọi mô hình này là "Mạng tính toán".

\section{MẠNG TÍNH TOÁN}

Mang tính toán là một biểu diễn tri thức có thể dùng để biểu diễn các tri thức về các vấn đề tính toán và được áp dụng một cách có hiệu quả để giải một số dạng bài toán. Mỗi mạng tính toán là một mạng ngữ nghĩa chứa các biến và những quan hệ có thể cài đặt và sử dụng được cho việc tính toán. Chúng ta xét một mạng tính toán gồm một tập hợp các biến cùng với một tập các quan hệ (chẳng hạn các công thức) tính toán giữa các biến. Trong ứng dụng cụ thể mối biến và giá trị của nó thường găn liền với một khái niệm cụ thể về sự vật, mỗi quan hệ thể hiện một tri thức về sự vật. 


\section{Các quan hệ}

Cho $M=\left\{x_{1}, x_{2}, \ldots, x_{m}\right\}$ là một tập hợp các biến có thể lấy giá trị trong các miền xác định tương ứng $D_{1}, D_{2}, \ldots, D_{m}$. Đối với mỗi quan hệ $R \subseteq D_{1} \times$ $D_{2} \times \cdots \times D_{m}$ trên các tập hợp $D_{1}, D_{2}, \ldots, D_{m}$ ta nói rằng quan hệ này liên kết các biến $x_{1}, x_{2}, \ldots, x_{m}$, và các ký hiệu là $R\left(x_{1}, x_{2}, \ldots, x_{m}\right)$ hay vắn tắt là $R(x)$ (ký hiệu $x$ dùng để chỉ bộ biến $\left\langle x_{1}, x_{2}, \ldots, x_{m}\right\rangle$ ). Ta có thễ thây rằng quan hệ $R(x)$ có thễ được biễu diễn bới một ánh xạ $f_{R, u, v}$ với $u \cup v=x$, và ta viết : $f_{R, u, v}: u \rightarrow v$, hay vắn tắt là $f: u \rightarrow v$.

Đối với các quan hệ dùng cho việc tính toán, cách ký hiệu trên bao hàm ý nghĩa như là một hàm: Ta có thể tính được giá trị của các biến thuộc $v$ khi biết được giá trị của các biến thuộc $u$.

Trong phần sau ta xét các quan hệ xác định bởi các hàm có dạng $f: u \rightarrow v$, trong đó $u \cap v=\emptyset$ (tập rỗng). Đặc biệt là các quan hệ dối xúng có hạng (rank) bằng một số nguyên dương $k$. Đó là các quan hệ mà ta có thể tính được $k$ biến bất kỳ từ $m-k$ biến kia (ớ đây $x$ là bộ gồm $m$ biến $\left\langle x_{1}, x_{2}, \ldots, x_{m}\right\rangle$ ). Ngoài ra, trong trường hợp cần nói rõ ta viết $u(f)$ thay cho $u, v(f)$ thay cho $v$. Đối với các quan hệ không phải là đối xứng có hạng $k$, không làm mất tính tông quát, ta có thể già sư quan hệ xác định duy nhất một hàm $f$ với tập biến vào là $u(f)$ và tập biến ra là $v(f)$; ta gọi loại quan hệ này là quan hệ không đối xứng và xác định một hàm, hay gọi vắn tắt là quan hệ không đối xứng.

Ví dụ: Quan hệ $f$ giữa 3 góc $A, B, C$ trong tam giác $A B C$ cho bởi hệ thức: $A+B+C=180$ (đơn vị: độ)

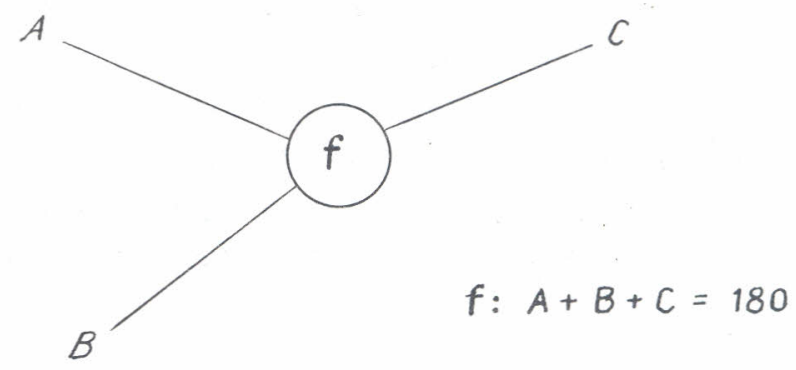

\section{Mạng tính toán và các ký hiệu}

Như đã nói trên, ta sẽ xem xét các mạng tính toán bao gồm một tập hợp các biến $M$ và một tập hợp các quan hệ $F$ trên biến. Trong trường hợp tông quát có thể viết:

$$
\begin{aligned}
M & =\left\{x_{1}, x_{2}, \ldots, n_{n}\right\}, \\
F & =\left\{f_{1}, f_{2}, \ldots, f_{m}\right\} .
\end{aligned}
$$

Đối với mỗi $f \in F$, ta ký hiệu $M(f)$ là tập các biến có liên hệ trong hệ $f$. Dĩ 
nhiên $M(f)$ là một tập con của $M: M(f) \subseteq M$. Nếu viết $f$ dưới dạng:

$$
f: u(f) \rightarrow v(f)
$$

thì ta có $M(f)=u(f) \cup v(f)$.

\section{BÀI TOÁN TRÊN MẠNG TÍNH TOÁN}

Cho một mạng tính toán $(M, F), M$ là tập các biến và $F$ là tập các quan hệ. Giả sứ có một tập biến $A \subseteq M$ đã được xác định và $B$ là một tập biến bất kỳ trong $M$. Các vấn đề đặt ra là:

1. Có thể xác định được tập $B$ từ tập $A$ nhờ các quan hệ trong $F$ hay không? Nói cách khác, ta có thể tính được giá trị của các biến thuộc $B$ với giả thiết đã biết giá trị của các biến thuộc $A$ hay không?

2. Nếu có thề xác định được $B$ từ $A$ thì quá trình tính toán giá trị của các biến thuộc $B$ như thế nào?

3. Trong trường hợp không thể xác định được $B$, thì cần cho thêm điều kiện gì để có thể xác định được $B$.

Bài toán xác định $B$ từ $A$ trên mạng tính toán $(M, F)$ được viết dưới dạng:

$$
A \rightarrow B
$$

trong ỏó $A$ được gọi là giả thiết, $B$ được gọi là mục tiêu tính toán của bài toán.

Đinh nghĩa 1. Bài toán $A \rightarrow B$ được goi là giải được khi có thể tính toán được giá trị các biến thuộc $B$ xuất phát từ giả thiết $A$. Ta nói rằng một dãy các quan hệ $\left\{f_{1}, f_{2}, \ldots, f_{k}\right\} \subseteq F$ là một lời giải của bài toán $A \rightarrow B$ nếu như ta lần lượt áp dụng các quan hệ $f_{i}(i=1, \ldots, k)$ xuất phát từ giả thiết $A$ thì sẽ tính được các biến thuộc $B$. Lời giải $\left\{f_{1}, f_{2}, \ldots, f_{k}\right\}$ được gọi là lời giải tốt nếu không thể bỏ bớt một số bước tính toán trong quá trình giải, tức là không thể bớt một số quan hệ trong lời giải.

Việc tìm lời giải cho bài toán là việc tìm ra một dãy quan hệ để có áp dụng suy ra được $B$ từ $A$. Điều này cũng có nghĩa là tìm ra được một quá trình tính toán để giải bài toán.

Định nghĩa 2. Cho $D=\left\{f_{1}, f_{2}, \ldots, f_{k}\right\}$ là một dãy quan hệ của mạng tính toán

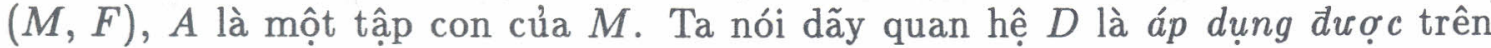
tập $A$ khi và chỉ khi ta có thể lần lượt áp dụng được các quan hệ $f_{1}, f_{2}, \ldots, f_{k}$ xuất phát từ giả thiết $A$.

Nhận xét: Trong định nghĩa trên, nếu đặt: $A_{0}=A, A_{1}=A_{0} \cup M\left(f_{1}\right), \ldots, A_{k}=$ $A_{k-1} \cup M\left(f_{k}\right)$, và ký hiệu $A_{k}$ là $D(A)$, thì ta có $D$ là một lời giải của bài toán 
$A \rightarrow D(a)$. Trong trường hợp $D$ là một dãy quan hệ bất kỳ (không nhất thiết là áp dụng được trên $\mathrm{A}$ ), ta vẩn ký hiệu $D(A)$ là tập biến đạt được khi lần lượt áp dụng các quan hệ trong dãy $D$ (nếu được). Chúng ta có thể nói rằng $D(A)$ là sự mở rộng của tập $A$ nhờ áp dụng dãy quan hệ $D$.

\section{GIẢI QUYÊTT VẤN ĐỀ}

\section{Tính giải được của bài toán}

Trong mục này chúng ta nêu lên một khái niệm có liên quan đến tính giải được của vấn đề trên một mạng tính toán: bao đóng của một tập hợp biến trên một mạng tính toán.

Định nghĩa 3. Cho mạng tính toán $(M, F)$ và $A$ là tập con của $M$. Ta có thể thấy rằng có duy nhất một tập hợp $B$ lớn nhất $\subseteq M$ sao cho bài toán $A \rightarrow B$ là giải được, và tập hợp $B$ này được gọi là bao đóng của $A$ trên mô hình $(M, F)$. Một cách trực quan, có thế nói bao đóng của $A$ là sự mở rộng tối đa của $A$ trên mồ hình $(M, F)$. Ký hiệu bao đóng của $A$ là $\bar{A}$ chúng ta có định lý sau đây:

Định lý 1. Trên một mạng tính toán $(M, F)$, bài toán $A \rightarrow B$ là giải aurợc khi và chì khi $B \subseteq \bar{A}$.

Từ định lý này, ta có thể kiểm tra tính giải được của bài toán $A \rightarrow B$ bằng cách tính bao đóng của tập $A$ rồi xem $B$ có bao hàm trong $\bar{A}$ hay không.

Định lý 2. Cho một mạng tính toán $(M, F), A, B$ là hai tập con của $M$. Ta có các điều sau dây là tương dương:

(1) $B \subseteq \bar{A}$.

(2) Có một dãy quan hệ $D=\left\{f_{1}, f_{2}, \ldots, f_{k}\right\} \subseteq F$ thóa các diều kiện:

(a) D áp được trên $A$.

(b) $D(A) \supseteq B$.

Chứng minh. Giả sử có (1), tức là $B \subseteq \bar{A}$. Khi đó bài toán là giải được. Do đó một dãy quan hệ $\left\{f_{1}, f_{2}, \ldots, f_{k}\right\} \subseteq F$ sao cho khi ta lần lượt áp dụng các quan hệ $f_{i}(i=1, \ldots, k)$ xuất phát từ giá thiết $A$ thì sẽ tính được các biến thuộc $B$. Dễ dàng thấy rằng dãy $\left\{f_{1}, f_{2}, \ldots, f_{k}\right\}$ này thỏa các điều kiện (2).

Đảo lại, giả sứ có $(2)$. Với các điều kiện có được bởi (2) ta thấy $\left\{f_{i}\right\}$ là lời giải của vấn đề $A_{i-1} \rightarrow A_{i}$, với mọi $i=1,2, \ldots, k$. Từ mệnh đề 2 suy ra bài toán $A_{0} \rightarrow A_{k}$ là giải được. Do đó bài toán $A \rightarrow B$ cũng giải được, suy ra $B \subseteq \bar{A}$ theo định lý 1 .

Qua các định lý trên, ta thấy rằng việc xác định bao đóng của một tập biến 
trên mô hình tính toán là cần thiết. Dưới đây là thuật toán cho phép xác định bao đóng của tập hợp $A \subseteq M$. Trong thuật toán này chúng ta thư áp dụng các quan hệ $f \in F$ dể tìm dần những biến thuộc $M$ có thể tính được từ $A$; cuối cùng sẽ được bao đóng của $A$.

Thuật toán 1. Tìm bao đóng cưa tập $A \subseteq M$ :

Nhập: Mạng tính toán $(M, F)$,

Xuất: $\bar{A}$

$$
A \subseteq M
$$

Thuât toán:

1. $B \leftarrow A$;

2. Repeat

$$
\begin{aligned}
& \begin{array}{l}
B 1 \leftarrow B \\
\text { for } f \in F \text { do } \\
\text { if ( } f \text { dối xứng and Card }(M(f) \backslash B) \leq r(f)) \text { or } \\
\\
\quad(f \text { không đối xứng and } M(f) \backslash B \subseteq v(f) \text { then } \\
\quad \text { begin } \\
\quad B \leftarrow B c u p M(f) ; \\
\quad F \leftarrow F \backslash\{f\} ; \| \text { loại } f \text { khói làn xem xét sau } \\
\text { end; }
\end{array} \\
& \text { Unil } B=B 1 ;
\end{aligned}
$$

3. $\bar{A} \leftarrow B$;

\section{Lời giải của bài toán}

Ơ trên ta đã nêu lên cách xác định tính giải được của bài toán. Tiếp theo, chúng ta sẽ trình bày cách tìm ra lời giải cho bài toán $A \rightarrow B$ trên mạng tính toán $(M, F)$.

Mệnh đề 1. Dãy quan hệ $D$ là một giải của bài toán $A \rightarrow B$ khi và chì khi $D$ áp dụng được trên $A$ và $D(A) \supseteq B$.

Do mệnh đề trên, để tìm một lời giải ta có thể làm như sau: Xuất phát từ giả thiết $A$, ta thử áp dụng các quan hệ để mở rộng dần tập các biển có giá trị được xác định; và quá trình này tạo ra một sự lan truyền tính xác định trên tập các biến cho đến khi đạt đến tập biến $B$. Dưới đây là thuật toán tìm một lời giải cho bài toán $A \rightarrow B$ trên mạng tính toán $(M, F)$.

Thuật toán 2. Tìm một lời giải cho bài toán $A \rightarrow B$ :

Nhâp: Mạng tính toán $(M, F)$, tập giả thiết $A \subseteq M$, tập biến cần tính $B \subseteq M$. 
Xuất: Lời giải cho bài toán $A t \leq B$.

Thuât toán:

1. Solution $\leftarrow$ empty; \| Solution là dãy các quan hệ sẽ áp dụng.

2. if $B \subseteq A$ then

begin

Solution_found $\leftarrow$ true: $\{$ biến Solution_found $=$ true $k$ hi bài toán là giải dược $\}$

goto 4 ;

end

else

Solution_found $\leftarrow$ false;

3. Repeat

$A_{\text {old }} \leftarrow A$;

Chọn ra một $f \in F$ chưa xem xét;

while not Solution_found and (chọn được $f$ ) do

begin

if ( $f$ dối xứng and $0<\operatorname{Card}(M(f) \backslash A) \leq r(f))$ or

$f$ không đối xứng and $\emptyset \neq M(f) \backslash A \subseteq v(f))$ then

begin

$A \leftarrow A \cup M(f)$;

Solution $\leftarrow$ Solution $\cup\{f\}$;

end;

if $B \subset A$ then

Solution_found $\leftarrow$ true;

Chọn ra một $f \in F$ chưa xem xét;

end; $\{$ while $\}$

Until Solution_found or $\left(A=A_{\text {old }}\right)$;

4. if not Solution_found then

Bài toán không có lời giải;

else

Solution là một lời giải.

Ghi chú:

1. Về sau, khi cần trình bày quá trình giải (hay bài giải) ta có thể xuất phát từ lời giải tìm được dưới dạng một dãy các quan hệ để xây dựng bài giải.

2. Lời giải (nếu có) tìm được trong thuật toán trên chưa chắc chắn là một lời giải tốt. Ta có thể bổ sung cho thuật toán ở trên thuật toán để tìm một lời giải tốt từ một lời giải đã biết nhưng chưa chắc là tốt. Thuật toán sẽ dựa trên định lý được trình bày tiếp theo đây. 
Định lý 3. Cho $D=\left\{f_{1}, f_{2}, \ldots f_{m}\right\}$ là một lời giải của bài toán $A \rightarrow B$. Úng với mỗi $i=1, \ldots, m$ đặt $D_{i}=\left\{f_{1}, f_{2}, \ldots f_{i}\right\}, D_{0}=\emptyset$. Ta xây dựng một họ các dãy con $S_{m}, S_{m-1}, \ldots, S_{2}, S_{1}$ của dãy $D$ nhu sau:

$$
\begin{array}{ll}
S_{m}=\emptyset & \text { nếu } D_{m-1} \text { là một lò̀i giải, } \\
f_{m}=\left\{f_{m}\right\} & \text { nếu } D_{m-1} \text { không là một lò̀i giải, } \\
S_{i}=S_{i+1} & \text { nếu } D_{i-1} \cup S_{i+1} \text { không là một lời giải, } \\
S_{i}=\left\{f_{i}\right\} \cup S_{i+1} & \text { nếu } D_{i-1} \cup S_{i+1} \text { không là một lời giải, } \\
\text { với mọi } i=m-1, m-2, \ldots, 2,1 .
\end{array}
$$

Khi đó ta có:

(1) $S_{m} \subseteq S_{m-1} \subseteq \cdots \subseteq S_{2} \subseteq S_{1}$.

(2) $D_{i-1} \cup S_{i}$ là một lời giải cúa bài toán $A \rightarrow B$ với mọi $i=m, \ldots, 2,1$.

(3) Nếu $S_{i}^{\prime}$ là một dãy con thật sự cưa $S_{i}$ thi $D_{i-1} \cup S_{i}^{\prime}$ không phải là một lờ $i$ giải cuia bài toán $A \rightarrow B$ với mọi $i$.

(4) $S_{1}$ là một lời giải tốt của bài toán $A \rightarrow B$.

Thuật toán 3. Tìm một lời giải tốt từ một lời giải đã biết.

Nhập: Mạng tính toán $(M, F)$,

lời giài $\left\{f_{1}, f_{2}, \ldots, f_{m}\right\}$ của bài toán $A \rightarrow B$.

Xuất: Lời giải tốt cho bài toán $A \rightarrow B$.

Thuât toán:

1. $D \leftarrow\left\{f_{1}, f_{2}, \ldots, f_{m}\right\}$;

2. for $i=m$ downto 1 do

if $D \backslash\left\{f_{i}\right\}$ là một lời giải then

$$
D \leftarrow D \backslash\left\{f_{i}\right\} ;
$$

3. $D$ là một lời giải tốt.

Trong thuật toán 3 có sử dụng việc kiểm tra một dãy quan hệ có phải là lời giải hay không. Việc kiểm tra này có thể được thực hiện nhờ thuật toán sau này:

\section{Thuật toán kiểm tra lời giải cho bài toán:}

Nhập: Mạng tính toán $(M, F)$, bài toán $A \rightarrow B$, dãy các quan hệ $\left\{f_{1}, f_{2}, \ldots, f_{m}\right\}$.

Xuất: Thông tin cho biết $\left\{f_{1}, f_{2}, \ldots, f_{m}\right\}$ có phải là lời giải của

bài toán $A \rightarrow B$ hay không.

Thuât toán:

1. for $i=1$ to $m$ do

if $\left(f_{i}\right.$ đối xứng and Card $\left(M\left(f_{i}\right) \backslash A\right) \leq r\left(f_{i}\right)$ or

( $f_{i}$ không đối xứng and $M\left(f_{i}\right) \backslash A \subseteq v\left(f_{i}\right)$ then $A \leftarrow A \cup M\left(f_{i}\right) ;$

2. if $A \supseteq B$ then $\left\{f_{1}, f_{2}, \ldots, f_{m}\right\}$ là lời giải else $\left\{f_{1}, f_{2}, \ldots, f_{m}\right\}$ khỗng là lời giải. 


\section{3. Định lý về sự phân tích quá trình giải}

Xét bài toán $A \rightarrow B$ trên mạng tính toán $(M, F)$. Trong mục này ta nêu lên một cách xây dựng quá trình giải từ một lời giải đã biết. Đối với một lời giải, rất có khả năng một quan hệ nào đó dẫn tới việc tính toán một số biến thừa, tức là các biến tính ra mà không có sử dụng các quan hệ trong lời giải và chỉ tính toán các biến thật sự cần thiết cho quá trình giải theo lời giải. Định lý sau đây cho ta một sự phân tích tập các biến được xác định theo lời giải và trên cơ sở đó có thể xây dựng quá trình tính toán các biến để giải quyết bài toán.

Định lý 4. Cho $\left\{f_{1}, f_{2}, \ldots, f_{m}\right\}$ là một lò̀i giải tốt cho bài toán $A \rightarrow B$ trên một mạng tính toán $(M, F)$. Đặt:

$$
A_{0}=A, A_{i}=\left\{f_{1}, f_{2}, \ldots\right\}(A) \text {, vớ } i \text { mọi } i=1, \ldots, m .
$$

Khi đó có một dãy $\left\{B_{0}, B_{1}, \ldots, B_{m-1}, B_{m}\right\}$, thóa các điều kiện sau dây:

(1) $B_{m}=B$.

(2) $B_{i} \subseteq A_{i}$, với mọi $i=0,1, \ldots, m$.

(3) Với mọi $i=1, \ldots, m,\left\{f_{i}\right\}$ là lời giải của bài toán $B_{i-1} \rightarrow B_{i}$ nhưng không phải là lời giải của bài toán $G \rightarrow B_{i}$, trong đó $G$ là một tập con thật sự tùy ý của $B_{i-1}$

Ghi chú:

1. Từ định lý trên ta có quá rình tính toán các biến để giải bài toán $A \rightarrow B$ như sau:

bước 1: tính các phép biến trong tập $B_{1} \backslash B_{0}$ (áp dụng $f_{1}$ ).

bước 2: tính các phép biến trong tập $B_{2} \backslash B_{1}$ (áp dụng $f_{2}$ ).

v.v...

bước $\mathrm{m}$ : tính các phép biến trong tập $B_{m} \backslash B_{m-1}$ (áp dụng $f_{m}$ ).

2. Từ chứng minh của định lý trên, ta có thể ghi ra một thuật toán để xây dựng dãy các tập biến $\left\{B_{1}^{\prime}, \ldots, B_{m-1}^{\prime}, B_{m}\right\}$ rời nhau cần lần lượt tính toán trong quá trình giải bài toán $\left(B_{i}^{\prime}=B_{i} \backslash B_{i-1}\right)$ gồm các bước chính như sau:

- xác định các tập $A_{0}, A_{1}, \ldots, A_{m}$.

- xác định các tập $B_{m}, B_{m-1}, \ldots, B_{1}, B_{0}$.

- xác định các tập $B_{1}^{\prime}, B_{2}^{\prime}, \ldots, B_{m}$.

\section{CÁC VÍ DỤ}

Trong phần này chúng ta nêu lên 2 ví dụ ứng dụng của mạng tính toán trong toán học và trong hóa học.

Ví dụ 1. Cho tam giác $\mathrm{ABC}$ có cạnh a và 2 góc kề là $\beta, \gamma$ được cho trước.

Tính diện tích $S$ của tam giác. 
Để tìm ra lời giải cho bài toán trước hết ta xét mạng tính toán của tam giác. Mạng tính toán này gồm:

1. Tập biến $M=\left\{a, b, c, \alpha, \beta, \gamma, h_{a}, h_{b}, h_{c}, S, p, R, r, \ldots\right\}$ trong đó $a, b, c$ là cạnh; $\alpha, \beta, \gamma$ là 3 góc tương ứng với $3 \mathrm{cạnh} ; h_{a}, h_{b}, h_{c}$ là 3 đường cao; $S$ là diện tích tam giác; $p$ là nưa chu vi; $R$ là bán kính đường tròn ngoại tiếp tam giác; $r$ là bán kính đường tròn nội tiếp tam giác, v.v...

2. Các quan hệ:

$$
\begin{array}{lll}
f_{1}: \alpha+\beta+\gamma=180 & f_{2}: \frac{a}{\sin \alpha}=\frac{b}{\sin \beta} & f_{3}: \frac{c}{\sin \gamma}=\frac{b}{\sin \beta} \\
f_{4}: \frac{a}{\sin \alpha}=\frac{c}{\sin \gamma} & f_{5}: p=(a+b+c) / 2 & f_{6}: S=a \cdot h_{a} / 2 \\
f_{7}: S=b . h_{b} / 2 & f_{8}: S=c . h_{c} / 2 & f_{9}: S=a . b \cdot \sin \gamma / 2 \\
\text { v.v... } & &
\end{array}
$$

3. Yêu cầu tính: $S$ (diện tích của tam giác).

Theo đề bài ta có giả thiết là: $A=\{a, \beta, \gamma\}$, và tập biến cần tính là $B=\{S\}$. Áp dụng thuật toán tìm lời giải (thuật toán 2 ) ta có một lời giải cho bài tính là dãy quan hệ sau: $\left\{f_{1}, f_{2}, f_{3}, f_{5}, f_{9}\right\}$. Xuất phát từ tập biến $A$, lần lượt áp dụng các quan hệ trong lời giải ta có tập các biến được xác định mở rộng dần đến khi $S$ được xác định:

$$
\begin{aligned}
\{a, \beta, \gamma\} \stackrel{f_{1}}{\longrightarrow} & \{a, \beta, \gamma, \alpha\} \stackrel{f_{2}}{\longrightarrow}\{a, \beta, \gamma, \alpha, b\} \stackrel{f_{3}}{\longrightarrow}\{a, \beta, \gamma, \alpha, b, c\} \\
& \stackrel{f_{5}}{\longrightarrow}\{a, \beta, \gamma, \alpha, b, c, p\} \stackrel{f_{9}}{\longrightarrow}\{a, \beta, \gamma, \alpha, b, c, p, S\}
\end{aligned}
$$

Có thể nhận thấy rằng lời giải này không phải là lời giải tốt vì có bước tính toán thừa, chẳng hạn là $f_{5}$. Thuật toán 3 sẽ lọc ra từ lời giải trên một lời giải tốt là $\left\{f_{1}, f_{2}, f_{9}\right\}$ :

$$
\{a, \beta, \gamma\} \stackrel{f_{1}}{\longrightarrow}\{a, \beta, \gamma, \alpha\} \stackrel{f_{2}}{\longrightarrow}\{a, \beta, \gamma, \alpha, b\} \stackrel{f_{9}}{\longrightarrow}\{a, \beta, \gamma, \alpha, b, S\}
$$

Theo lời giải trên, ta có quá trình tính toán như sau:

Bước 1: tính $\alpha$ (áp dụng $f_{1}$ ).

Bước 2: tính b (áp dụng $f_{2}$ ).

Bước 3: tính $S$ (áp dụng $f_{9}$ ).

Quá trình tính toán (gồm 3 bước) này có thể được diễn đạt một cách rõ ràng trên hình 1. 


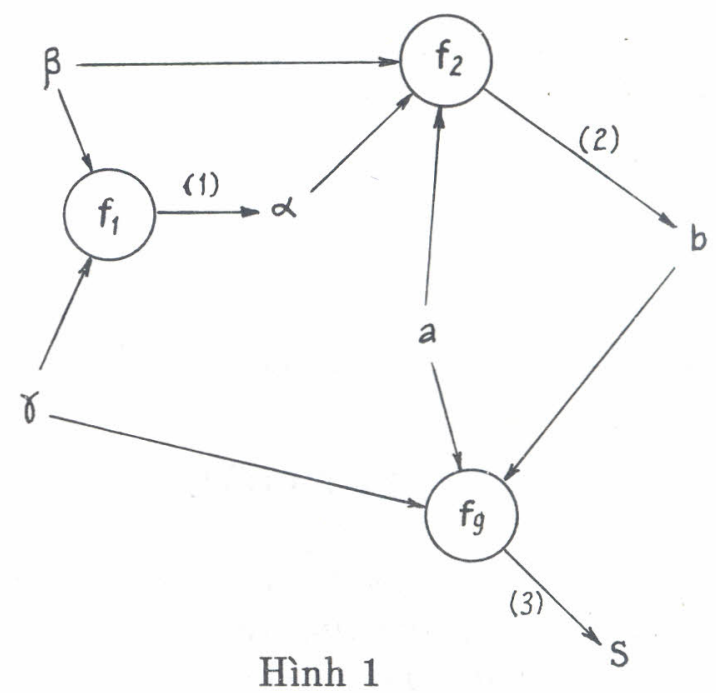

Ví dụ 2. Chúng ta biết rằng trong hóa học, việc xem xét phản ứng là một trong những vấn đề quan trọng. Về mặt tri thức người ta đã biết được nhiều chất và các phản ứng hóa học có thể chuyển hóa từ một số chất này thành các chất khác. Tạm thời bỏ qua một số điều kiện phản ứng, ta có thể xem tri thức đó như một mạng tính toán mà mối phản ứng là một quan hệ của mạng. Ví dụ như phản ứng điều chế çlo từ axít clohidric và đioxit mangan:

$$
\mathrm{MnO}_{2}+\mathrm{HCl} \rightarrow \mathrm{MnCl}_{2}+\mathrm{Cl}_{2} \uparrow+\mathrm{H}_{2} \mathrm{O}
$$

Phản ứng trên có thể được xem như một quan hệ cho chúng ta có được các chất $\mathrm{Cl}_{2}, \mathrm{MnCl}_{2}, \mathrm{H}_{2} \mathrm{O}$ từ các chất $\mathrm{MnO}_{2}, \mathrm{HCl}$.

Giả sử ta biết một số những phản ứng hóa học. Chằng hạn như một phản ứng của clo sau đây:

$$
\begin{aligned}
& \mathrm{Na}+\mathrm{Cl}_{2} \rightarrow \mathrm{NaCl} \\
& \mathrm{Fe}+\mathrm{Cl}_{2} \rightarrow \mathrm{FeCl}_{3} \\
& \mathrm{Cu}+\mathrm{Cl}_{2} \rightarrow \mathrm{CuCl}_{2} \\
& \mathrm{Cl}_{2}+\mathrm{H}_{2} \mathrm{O} \rightarrow \mathrm{HCl}+\mathrm{HClO} \\
& \mathrm{MnO}_{2}+4 \mathrm{HCl}=\mathrm{MnCl}_{2}+\mathrm{Cl}_{2} \uparrow+2 \mathrm{H}_{2} \mathrm{O} \\
& \mathrm{HCl}+\mathrm{KMnO}_{4} \rightarrow \mathrm{KCl}+\mathrm{MnCl}_{2}+\mathrm{H}_{2} \mathrm{O}+\mathrm{Cl}_{2} \uparrow \\
& \mathrm{NaCl}+\mathrm{H}_{2} \mathrm{O} \rightarrow \mathrm{Cl}_{2} \uparrow+\mathrm{H}_{2} \uparrow+\mathrm{NaOH} \\
& \mathrm{K}+\mathrm{Cl}_{2} \rightarrow \mathrm{KCl}
\end{aligned}
$$

Bây giờ giả sử ta phải giải quyết bài toán điều chế như sau: Cho rằng ta có các chất hóa học $\mathrm{S}, \mathrm{H}_{2} \mathrm{O}, \mathrm{NaCl}$; hãy tìm các phản ứng điều chế các chất hóa học $\mathrm{Na}_{2} \mathrm{SO}_{4}, \mathrm{H}_{2} \mathrm{SO}_{4}, \mathrm{HCl}, \mathrm{Na}$.

Bằng các tiến hành thuật giải tìm lời giải nêu trong phần IV.2 ờ trên trong tập các phản ứng hóa học ta có thễ tìm ra các phản ứng sau đây: 


$$
\begin{aligned}
& \mathrm{H}_{2} \mathrm{O}+\mathrm{NaCl} \rightarrow \mathrm{NaOH}+\mathrm{H}_{2}+\mathrm{Cl}_{2} \\
& \mathrm{H}_{2}+\mathrm{Cl}_{2} \rightarrow \mathrm{HCl} \\
& \mathrm{H}_{2} \mathrm{O} \rightarrow \mathrm{O}_{2}+\mathrm{H}_{2} \\
& \mathrm{NaCl} \rightarrow \mathrm{Cl}_{2}+\mathrm{Na} \\
& \mathrm{S}+\mathrm{O}_{2} \rightarrow \mathrm{SO}_{2} \\
& \mathrm{SO}_{3}+\mathrm{H}_{2} \mathrm{O} \rightarrow \mathrm{H}_{2} \mathrm{SO}_{4} \\
& \mathrm{H}_{2} \mathrm{SO}_{4}+\mathrm{NaOH} \rightarrow \mathrm{Na}_{2} \mathrm{SO}_{4}+\mathrm{H}_{2} \mathrm{O}
\end{aligned}
$$

\section{KẾT LUẬN}

Trên đầy chúng tôi đã nêu lên mô hình rất hữu dụng cho việc giải tự động một số dạng bài toán: mô hình mạng tính toán. Một số thuật toán giải một số vấn đề trên mô hình đã được trình bày và chứng minh chặt chẽ về mặt lý thuyết. Hai ví dụ ứng dụng mô hình mạng tính toán được nêu trong bài cũng đã được cài đặt và cho chạy thử. Chương trình cho lời giải tốt trong mọi trường hợp. Mô hình này nếu được nghiên cứu và phát triển thêm nữa sẽ trở thành một công cự rất tốt cho việc thiết kế và cài đặt các hệ giải bài toán tự động.

\section{TÀI LIỆU THAM KHẢO}

1. Kỷ yểu Hội nghị khoa học: "Công nghệ thông tin: Nghiên cứu và phát triển", Viện Công nghệ thông tin, tháng 12, 1996.

2. Enn Tyugu, Knowledge-based Programming. Addison-Wesley Publishing company, 1988.

3. Elaine Rich \& Kevin Knight, Artificial Intelligence. McGraw-Hill, Inc, 1991.

4. Jean-Louis Laurière, Problem-Solving and Artificcial Intelligence, Pretice Hall, 1990.

5. Bạch Hưng Khang \& Hoàng Miếm, Trí tuệ nhân tạo, các phuơng pháp và úng dụng. Nhà Xuất bản Khoa học và Kỹ thuật, Hà Nội, 1989.

6. Hojjat Adeli, Knowlegde Engineering, Vol I. mcGraw-Hill, Inc, 1990.

7. Hojjat Adeli, Knowlegde Engineering, Vol II. mcGraw-Hill, Inc., 1990.

8. Judea Pearl, Heuristics. Addoson-Wesley Publishing company, 1984.

9. J. D. Ulman, Principles of Database and Knowlegde-base Systems, Vol I. Computer Science Press, 1988.

10. J. D. Ullman, Principles of Database and Knowledge-base Systems Vol II. Computer Science Press, 1989.

Viện Công nghệ thông tin

Trung tâm KHTN và CNQG

Nhận bài ngày 15-4-1997 